\title{
Мощность насыщения оптического усилителя на основе самоорганизующихся квантовых точек
}

\author{
(C) А.Е. Жуков ${ }^{1}$, Н.В. Крыжановская ${ }^{1}$, Э.И. Моисеев ${ }^{1}$, А.М. Надточий ${ }^{1}$, Ф.И. Зубов ${ }^{2}$, \\ М.В. Фетисова ${ }^{2}$, М.В. Максимов ${ }^{2,1}$, Н.Ю. Гордеев ${ }^{3}$ \\ ${ }^{1}$ Национальный исследовательский университет „Высшая школа экономики“, \\ 190008 Санкт-Петербург, Россия \\ ${ }^{2}$ Санкт-Петербургский национальный исследовательский Академический университет им. Ж.И. Алфёрова \\ Российской академии наук, \\ 194021 Санкт-Петербург, Россия \\ ${ }^{3}$ Физико-технический институт им. А.Ф. Иофффе Российской академии наук, \\ 194021 Санкт-Петербург, Россия \\ E-mail: zhukale@gmail.com
}

Поступила в Редакцию 22 апреля 2021 г.

В окончательной редакции 28 апреля 2021 г.

Принята к публикации 28 апреля 2021 г.

На основе анализа скоростных уравнений с помощью численного моделирования и аналитически исследовано насыщение усиления в оптическом усилителе на основе массива квантовых точек. Показано, что при умеренном уровне инжекции величина мощности насыщения растет пропорционально плотности тока, а в дальнейшем достигает своего наибольшего значения, ограниченного скоростью поступления носителей заряда на основное состояние и количеством квантовых точек, взаимодействующих с фотонами. Предложены выражения, позволяющие в явном виде описать зависимость мощности насыщения от тока и ее взаимосвязь с внутренними параметрами активной области.

Ключевые слова: полупроводниковый оптический усилитель, квантовые точки, скоростные уравнения.

DOI: 10.21883/FTP.2021.09.51302.9669

\section{1. Введение}

Самоорганизующиеся квантовые точки (КТ) доказали свою эффективность в качестве активной области низкопороговых инжекционных лазеров [1], микродисковых лазеров [2,3], лазеров [4] и микролазеров [5,6], монолитно интегрированных с кремнием, а также лазеров, работающих в режиме синхронизации мод [7]. Еще одной активно развивающейся областью применения КТ является создание на их основе полупроводниковых оптических усилителей (ОУ) [8]. Достоинствами КТ $\mathrm{InAs} / \mathrm{InGaAs} / \mathrm{GaAs}$ является возможность достижения высоких значений коэффициента усиления в широком (> 100 нм [9]) спектральном диапазоне вблизи длины волны 1.3 мкм, соответствующей близкой к нулю хроматической дисперсии в стандартном одномодовом оптическом волокне. Это открывает перспективы применения ОУ на основе КТ в оптических интегральных схемах, использующих спектральное уплотнение каналов. Теоретическое исследование КТ ОУ в основном затрагивало динамические эффекты [10-12]. В то же время в литературе практически отсутствуют работы [13], посвященные анализу статических характеристик КТ ОУ, более важных с точки зрения применения ОУ совместно с лазерами, работающими в непрерывном режиме.

Наиболее значимой характеристикой является мощность насыщения $P_{S}[14]$, при которой коэффициент усиления оптического сигнала в стационарном режиме снижается в 2 раза по отношению к своему малосигнальному значению. Снижение усиления в полупроводниковом
ОУ при высоких уровнях входной оптической мощности связано с уменьшением концентрации носителей заряда в активной области вследствие их рекомбинации, стимулированной большой плотностью фотонов в резонаторе. Упрощенное рассмотрение свойств полупроводникового ОУ, основанное на предположении линейной связи усиления и концентрации носителей [14], предсказывает не зависящую от тока инжекции величину мощности насыщения, обратно пропорциональную дифференциальному усилению. Особенностью КТ является насыщение усиления [15], выражающееся в быстром замедлении зависимости усиления от плотности тока накачки. Таким образом, с ростом заполнения КТ дифференциальное усиление снижается, что должно вести к увеличению $P_{S}$ при больших плотностях инжекции. В цитированной выше работе [13] было выполнено численное моделирование, показавшее возможность достижения с помощью КТ большой мощности насыщения (> 20 дБм при плотности тока $\left.10 \kappa \mathrm{A} / \mathrm{cm}^{2}\right)$, приблизительно на порядок превосходящих значения, характерные для ОУ на основе квантовой ямы. Было отмечено, что мощность насыщения возрастает с током накачки усилителя. Однако аналитическое выражение, позволяющее в явном виде оценить величину $P_{S}$, получено не было, что затрудняет возможности как анализа приборных характеристик КТ OУ, так и поиск путей их дальнейшей оптимизации.

В настоящей работе нами выполнен анализ оптического усиления в режиме больших плотностей тока инжекции и уровней оптической мощности для модельной плотности состояний массива квантовых точек и полу- 
чены простые аналитические выражения для величины мощности насыщения усиления.

\section{2. Скоростные уравнения КТ ОУ}

Система скоростных уравнений, использованная нами для анализа КТ ОУ, близка ранее использованной при рассмотрении лазеров на основе КТ [16-18]. Она основана на предположении захвата носителей заряда на основное состояние КТ из окружающей их матрицы в результате каскада переходов с верхнего (возбужденного) уровня КТ на нижележащий уровень. Такой каскадный характер захвата был экспериментально подтвержден в работе [19]. Мы рассматриваем основное (0) и два возбужденных $(1,2)$ состояния КТ, а также резервуap (R), поставляющий носители заряда в КТ (рис. 1). $\mathrm{B}$ установившемся режиме имеет место баланс между поступлением носителей заряда в результате инжекции или захвата с вышележащего уровня и их уходом с уровня вследствие спонтанной рекомбинации, захвата на нижележащий уровень и выброса на вышележащий уровень. Для основного уровня также имеет место уход носителей заряда вследствие вынужденной рекомбинации.

Оптическая мощность распределена вдоль оси оптического усилителя неоднородно. Рассмотрим фрагмент оптического усилителя, длина которого достаточно мала, чтобы полагать в его пределах плотность фотонов постоянной. Система скоростных уравнений имеет вид:

$$
\begin{gathered}
\frac{J}{e}+\frac{n_{2} f_{2}\left(1-f_{R}\right)}{\tau_{2-\mathbf{R}}}=\gamma_{R} n_{R} f_{R}^{2}+\frac{n_{R} f_{R}\left(1-f_{2}\right)}{\tau_{R-2}}, \\
\frac{n_{\mathrm{R}} f_{R}\left(1-f_{2}\right)}{\tau_{\mathrm{R}-2}}+\frac{n_{1} f_{1}\left(1-f_{2}\right)}{\tau_{1-2}} \\
\quad=\gamma_{2} n_{2} f_{2}^{2}+\frac{n_{2} f_{2}\left(1-f_{1}\right)}{\tau_{2-1}}+\frac{n_{2} f_{2}\left(1-f_{\mathrm{R}}\right.}{\tau_{2-R}}, \\
\frac{n_{2} f_{2}\left(1-f_{1}\right)}{\tau_{2-1}}+\frac{n_{0} f_{0}\left(1-f_{1}\right)}{\tau_{0-1}} \\
=\gamma_{1} n_{1} f_{1}^{2}+\frac{n_{1} f_{1}\left(1-f_{2}\right)}{\tau_{1-2}}+\frac{n_{1} f_{1}\left(1-f_{0)}\right.}{\tau_{1-0}}, \\
\frac{n_{1} f_{1}\left(1-f_{0}\right)}{\tau_{1-0}}=\gamma_{0} n_{0} f_{0}^{2}+\frac{n_{0} f_{0}\left(1-f_{1}\right)}{\tau_{0-1}}+G \frac{p}{\varepsilon} .
\end{gathered}
$$

Здесь $J$ - плотность тока инжекции; $n_{i}-$ эффективная листовая плотность состояний $(i=0,1,2, \mathrm{R})$, которая для КТ определяется произведением полной листовой плотности КТ $\left(n_{\mathrm{QD}}\right)$ и степени вырождения уровня $\left(\kappa_{i}\right) ; f_{i}-$ степень заполнения; $\gamma_{i}-$ темп спонтанной рекомбинации; $\tau_{i-j}-$ время захвата с $i$-го на $j$-й уровень и связанное в ним время выброса $\tau_{j-i}=\tau_{i-j}\left(n_{j} / n_{i}\right) \exp \left(\Delta_{i-j}\right)$, где $\Delta_{i-j}-$ разница по энергии между уровнями, отнесенная к величине тепловой энергии; $p$ - отнесенная к ширине полоска световая мощность фотонов с энергией $\varepsilon$, соответствующей основному переходу; $G=G_{\max }\left(2 f_{0}-1\right)-$

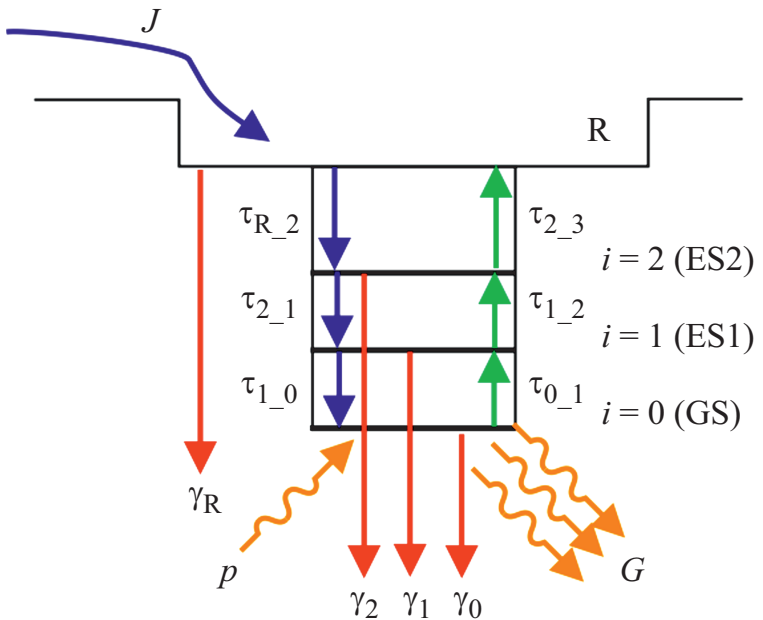

Рис. 1. Схематическое изображение энергетической структуры и основных процессов, протекающих в КТ ОУ.

модовое оптическое усиление на основном переходе, достигающее своего наибольшего значения $G_{\max }$ при полном заселении основного уровня КТ.

Система уравнений (1)-(4) базируется на следующих допущениях. Мы полагаем, что все КТ могут взаимодействовать с фотонами рассматриваемой энергии $\varepsilon$, т.е. иными словами, однородное уширение оптического перехода КТ больше или по крайней мере сопоставимо с неоднородным уширением плотности состояний ансамбля КТ. Хотя это соотношение, как правило, не отвечает реальной ситуации, подобное допущение значительно упрощает анализ и потому широко используется, в том числе в цитированных выше работах [16-18]. Далее мы обсудим, каким образом может быть учтено однородное уширение. Также мы полагаем, что электроны и дырки характеризуются одинаковыми параметрами, что ведет, в частности, для любого уровня к равенству степени его заполнения электронами и степени заполнения дырками. В реальности дырочные уровни могут, по сравнению с электронными, характеризоваться меньшим энергетическим разделением и более длинными временами захвата на них, что ведет к некоторым особенностям поведения КТ в режиме одновременной генерации через основной и первый возбужденный оптические переходы [20,21]. Однако для работы ОУ данное обстоятельство не является существенным, поскольку, как будет показано далее, мощность насыщения определяется максимальным усилением, и, таким образом, неэквивалентность электронов и дырок может быть учтена через свое влияние на величину $G_{\max }$. Наконец, мы полагаем, что эффективная плотность состояний резервуара достаточно велика, так что вплоть до наибольших рассматриваемых плотностей тока его заполнение оставалось заметно меньше единицы. Отказ от этого допущения потребует интегрирования зависящей от энергии плотности состояний, что не приведет к каким-либо существенным последствиям с точки зрения интересующих нас явлений, но значительно усложнит численный счет. 
Для численного решения системы скоростных уравнений из (1) степень заполнения 2-го возбужденного состояния КТ $f_{2}$ может быть выражена как функция заполнения резервуара $f_{R}$ и плотности тока $J$. Затем последовательно находим $f_{1}$ из (2) и $f_{0}$ из (3), после чего из (1) получаем трансцендентное уравнение, описывающее в неявном виде зависимость степеней заполнения состояний от плотности тока и мощности. Окончательно для некоторой плотности тока находим являющуюся целью нашего анализа внутреннюю мощность насыщения $p_{S}$ из уравнения $G\left(p_{S}, J\right)=G(0, J) / 2$. Поскольку плотность тока остается неизменной вдоль оси ОУ, он характеризуется одинаковым значением $p_{S}$ во всех своих частях независимо от конкретного вида взаимосвязи $p_{S}(J)$. Это позволяет при анализе приборных характеристик КТ ОУ использовать хорошо известные [14] интегральные соотношения и, в частности, определить выходную мощность $p_{\text {Sout }}$, при которой интегральный коэффициент усиления ОУ спадает в 2 раза по отношению к своему значению $A_{0}$ при низком уровне входного сигнала как $p_{\text {Sout }}=\ln (2) p_{S} A_{0} /\left(A_{0}-2\right)$.

При расчетах нами был использован следующий базовый набор параметров:

$$
\begin{gathered}
n_{Q D}=5 \cdot 10^{11} \mathrm{~cm}^{-2}, \quad G_{\max }=40 \mathrm{~cm}^{-1}, \quad \kappa_{0}=2, \\
\kappa_{2}: \kappa_{1}: \kappa_{0}=5: 3: 1, \quad n_{R}=2 \cdot 10^{14} \mathrm{~cm}^{-2}, \\
\tau_{R-2}=\tau_{2-1}=\tau_{1-0}=3 \Pi \mathrm{c}, \quad \gamma_{R}=2 \cdot 10^{9} \mathrm{c}^{-1}, \\
\gamma_{2}=\gamma_{1}=\gamma_{0}=1 \cdot 10^{9} \mathrm{c}^{-1}, \Delta_{R-2}=3, \Delta_{2-1}=\Delta_{1-0}=1.5 .
\end{gathered}
$$

\section{3. Результаты численного моделирования}

На рис. 2 показаны степень заполнения основного состояния КТ и усиление на основном оптическом переходе в зависимости от оптической мощности при

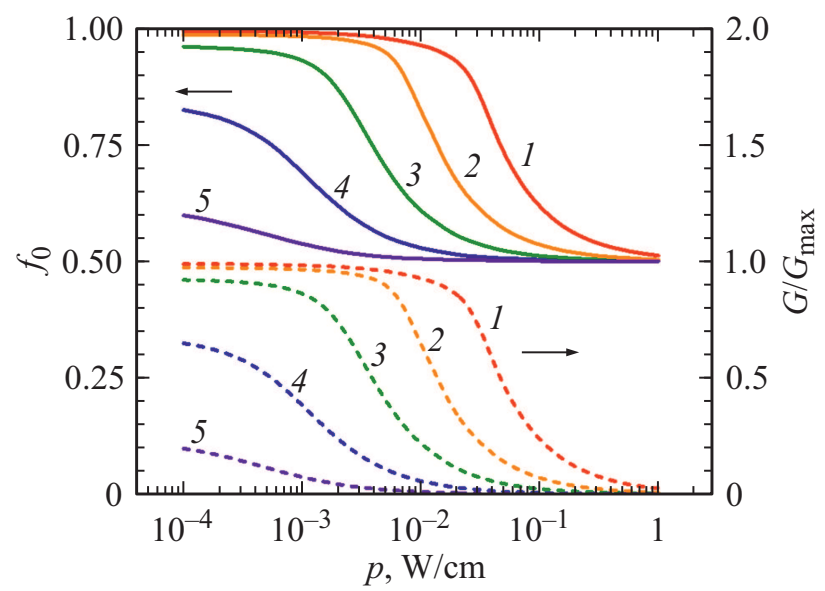

Рис. 2. Зависимость заполнения основного состояния (сплошные линии) и оптического усиления (штриховые линии) от плотности оптической мощности при различном токе инжекции: $1-0.1,2-0.3,3-1,4-3,5-10 \kappa \mathrm{A} / \mathrm{cm}^{2}$.
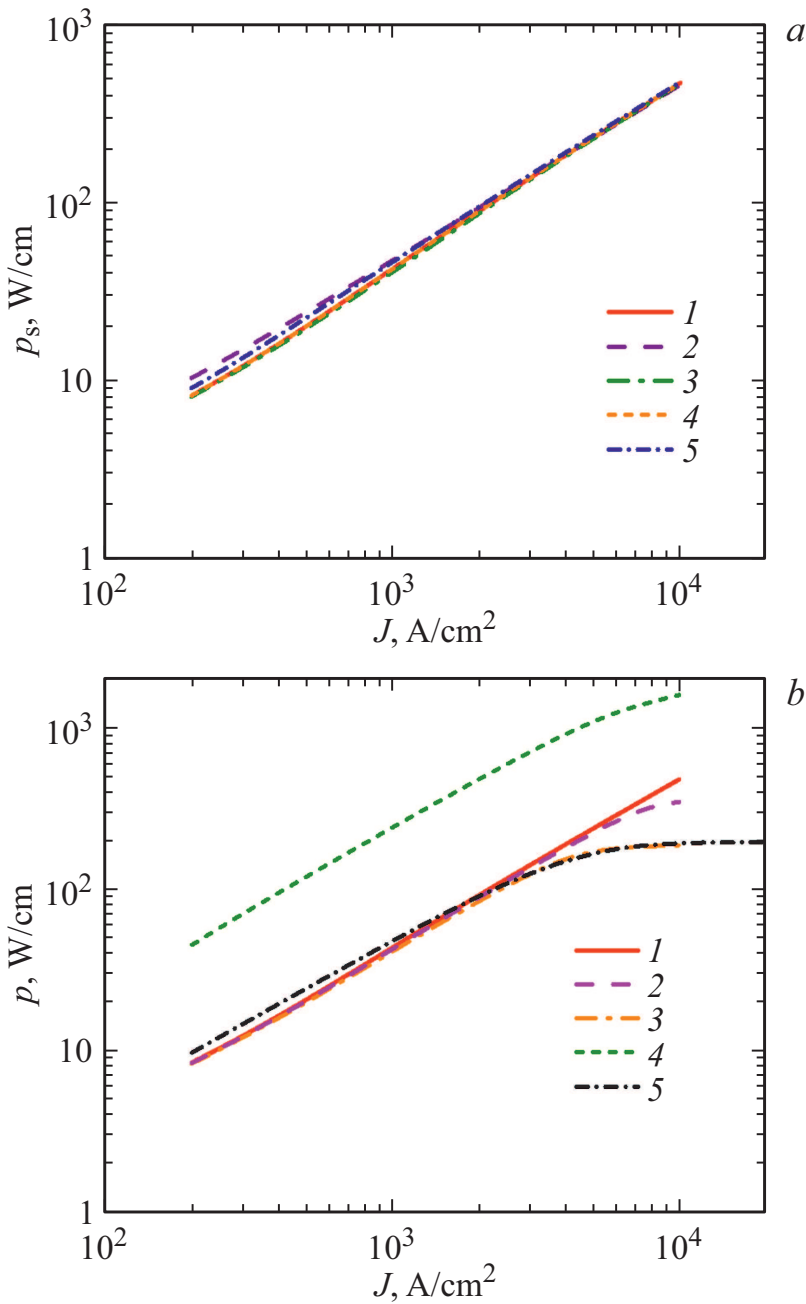

Рис. 3. Результаты численного моделирования зависимости мощности насыщения усиления от плотности тока для исходного набора параметров $(1)$, а также при изменении значения одного из параметров: $(a) 2-\kappa_{2}=50,3-\Delta_{R-2}=5,4-$ $\tau_{R-2}=15 \Pi \mathrm{c}, 5-n_{R}=2 \cdot 10^{15} \mathrm{~cm}^{-2} ;$ (b) $2-\tau_{1-0}=15$ пс, $3-\tau_{1-0}=30$ пс, $4-n_{Q D}=1 \cdot 10^{11} \mathrm{~cm}^{-2}, 5-$ расчет с помощью (8). (Цветной вариант рисунка представлен в электронной версии статьи).

различных значениях плотности тока инжекции. При низком уровне оптической мощности заполнение основного уровня с ростом тока инжекции быстро возрастает и уже начиная с $1 \mathrm{\kappa A} / \mathrm{cm}^{2}$ становится близким к 1. Соответственно, усиление оказывается близко к своему максимальному значению, $G \approx G_{\max }$. Увеличение мощности ведет к подавлению заполнения уровня до $1 / 2$ и снижению усиления до 0 . Как видно, чем больше плотность тока, тем при более высоком уровне мощности падение усиления становится заметным. Это означает, что мощность насыщения усиления не остается постоянной, но растет с ростом тока.

На рис. 3, $a$ представлена зависимость мощности насыщения усиления от плотности тока. В исследованном диапазоне инжекции и использованного при расчетах 
набора параметров эта зависимость является линейной функцией, причем на нее практически не оказывает влияние изменение величины следующих параметров: увеличение степени вырождения одного из возбужденных состояний, увеличение глубины локализации КТ и времени захвата носителей из резервуара, а также плотность состояний резервуара. Результаты расчетов, соответствующие этим случаям, также приведены на рис. $3, a$.

Мы нашли, что существенным оказывается изменение времени перехода носителей с 1-го возбужденного уровня КТ на основной уровень $\tau_{1-0}$, а также листовой плотности массива КТ (рис. $3, b$ ). В последнем случае мы полагали, что максимальное усиление меняется пропорционально $n_{Q D}$. Увеличение времени захвата не вызывает изменения зависимости $p_{S}(J)$ вплоть до самых высоких уровней инжекции, при которых величина мощности насыщения перестает расти, достигая своего предельного значения $p_{S \max }$. Рост $\tau_{1-0}$ ведет к пропорциональному снижению $p_{S \max }$, а также уменьшению плотности тока, $J_{\max }$, начиная с которой величина $p_{S}$ практически перестает меняться. Уменьшение плотности квантовых точек вызывает пропорциональное увеличение коэффициента пропорциональности, описывающего линейную зависимость $p_{S}(J)$, т. е. при умеренных значениях тока инжекции. В области больших токов становится заметным тенденция к прекращению роста $p_{S}$.

\section{4. Асимптотики и аналитические выражения}

Имея в виду задачи оптимизации ОУ, например выбора оптимальной конструкции массива квантовых точек или рабочего тока, было бы весьма полезно получить простые аналитические выражения, способные описать зависимость насыщения от плотности тока инжекции и взаимосвязь этой зависимости с параметрами активной области. При низком уровне оптической мощности практически для всех значений плотности тока накачки $G \approx G_{\max }$. Таким образом, мощность насыщения может быть приближенно определена из условия $G\left(p_{S}\right) \approx G_{\max } / 2$. Из (1)-(4) мы можем получить следующее выражение: $J / e=\gamma_{R} n_{R} f_{R}^{2}+\gamma_{2} n_{2} f_{2}^{2}$ $+\gamma_{1} n_{1} f_{1}^{2}+\gamma_{0} n_{0} f_{0}^{2}+G p / \varepsilon$, которое показывает, что ток инжекции расходуется на рекомбинацию носителей заряда, тогда как переходы носителей между уровнями не ведут к потреблению тока. При больших плотностях оптической мощности, вызывающих насыщение усиления, слагаемое, связанное со стимулированной рекомбинацией, преобладает над прочими, так что $J / e \approx G p / \varepsilon \approx\left(G_{\max } / 2\right)\left(p_{S} / \varepsilon\right)$, откуда мы получаем оценку:

$$
p_{S} \approx \frac{2 \varepsilon J}{e G_{\max }} .
$$

При дальнейшем увеличении плотности тока заполнение 1-го возбужденного уровня КТ возрастает

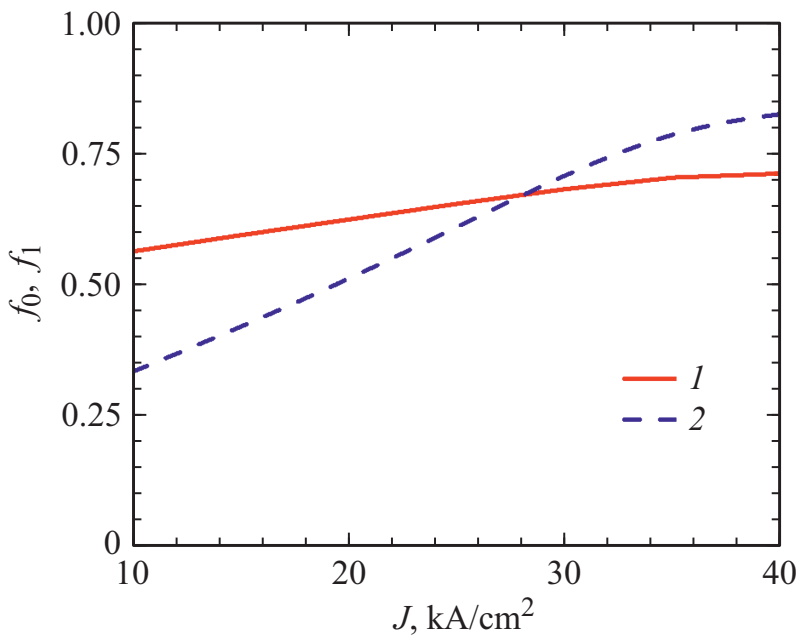

Рис. 4. Зависимость степени заполнения состояний КТ от плотности тока при плотности мощности 1.9 кВт/см $\left(p_{S \max }\right)$ : $1-f_{0}, 2-f_{1}$.

и начинает превосходить заполнение основного уровня (рис. 4), поскольку носители с основного состояния быстро удаляются вследствие стимулированной рекомбинации. Приближенно можно полагать, что $f_{1}$ близко к 1 , тогда как $f_{0}$ при достижении мощности насыщения составляет $3 / 4$, что соответствует усилению $G_{\max } / 2$. Тогда из $(4)$ следует $n_{1} /\left(4 \tau_{1-0}\right) \approx$ $\approx \gamma_{0} n_{0} f_{0}^{2}+\left(G_{\max } / 2\right)\left(p_{S} / \varepsilon\right) \approx\left(G_{\max } / 2\right)\left(p_{S} / \varepsilon\right)$, откуда получаем оценку для наибольшего уровня мощности насыщения, достигаемого при увеличении тока инжекции:

$$
p_{S} \approx p_{S \max }=\frac{\varepsilon \kappa_{1} n_{Q D}}{\tau_{1-0} G_{\max }} .
$$

Для обобщения асимптотического поведения зависимости $p_{S}(J)$ может быть предложено одно из следующих выражений:

$$
\begin{gathered}
p_{S} \approx p_{S \max } \text { th }\left(\frac{J}{J_{S \max }}\right), \\
p_{S} \approx \frac{p_{S \max }}{\sqrt{1+\left(\frac{J}{J_{S \max }}\right)^{2}}},
\end{gathered}
$$

оба из которых описывают линейное возрастание усиления насыщения в области умеренных токов в соответствии с (5) и последующее достижение своего предельного значения (6). Излом зависимости происходит при плотности тока

$$
J_{S \max }=\frac{e G_{\max } p_{S \max }}{2 \varepsilon}=\frac{e \kappa_{1} n_{Q D}}{2 \tau_{1-0}},
$$

которая отвечает приблизительно одному акту стимулированного испускания фотона каждой квантовой точкой в промежуток времени, соответствующий захвату носителя заряда на основное состояние. Можно говорить, что при токах, меньших $J_{S \max }$, величина усиления в области больших плотностей фотонов ограничена темпом 
инжекции, а при токах, превышающих $J_{S \max }$, ограничена темпом захвата на нижний уровень.

Полученные аналитические выражения позволяют произвести оценку насыщенного усиления с учетом большого неоднородного уширения плотности состояний ансамбля КТ. В подобных условиях фотоны способны взаимодействовать не со всеми КТ, а лишь с теми, чьи энергии основного оптического перехода лежат по отношению к энергии фотонов в пределах величины однородного уширения. Это ведет к соответствующему уменьшению плотности КТ в выражениях (7) и (10), что в свою очередь вызовет пропорциональное снижение предельной мощности насыщения усиления и плотности тока, отвечающей прекращению роста величины $p_{S}$. Так, полагая, что с фотонами взаимодействует лишь 20\% ансамбля на основе 10 рядов КТ, оценка $p_{S \max }$ дает 380 Вт/см или 190 мВт (23 дБм) для полоска шириной 5 мкм. Плотность тока, отвечающая переходу в режим ограничения скоростью захвата, составит при этом 8 кА/см2.

\section{5. Заключение}

Таким образом, скоростные уравнения, ранее использованные для описания поведения лазера на основе квантовых точек, были применены для моделирования характеристик оптического усилителя. Выполненные расчеты показывают, что для набора параметров, который примерно соответствует характеристикам массивов квантовых точек InAs/InGaAs/GaAs, мощность насыщения усиления линейно растет с током накачки по крайней мере до плотностей тока $10 \kappa \mathrm{A} / \mathrm{cm}^{2}$. Более медленный захват носителей заряда на основное состояние или уменьшение плотности массива КТ ведет к тому, что наблюдается прекращение роста мощности насыщения в зависимости от тока. В этом режиме усиление квантовых точек становится ограниченным скоростью поступления носителей заряда на основной уровень. Предложенное аналитическое выражение позволяет описать значение мощности насыщения в обоих режимах и предсказать ее изменение при варьировании параметров массива КТ, например, вследствие неоднородного уширения плотности состояний.

\section{Финансирование работы}

Работа выполнена при поддержке проекта РНФ 19-72-30010. Компьютерные расчеты выполнены в рамках Программы фундаментальных исследований НИУ ВШЭ.

\section{Конфликт интересов}

Авторы заявляют, что у них нет конфликта интересов.

\section{Список литературы}

[1] D.G. Deppe, K. Shavritranuruk, G. Ozgur, H. Chen, S. Freisem. Electron. Lett., 45, 54 (2009).

[2] M.-H. Mao, H.-C. Chien, J.-Z. Hong, C.-Y. Cheng. Opt. Express, 19, 14145 (2011).

[3] N.V. Kryzhanovskaya, E.I. Moiseev, Yu.V. Kudashova, F.I. Zubov, A.A. Lipovskii, M.M. Kulagina, S.I. Troshkov, Yu.M. Zadiranov, D.A. Livshits, M.V. Maximov, A.E. Zhukov. Electron. Lett., 51, 1354 (2015).

[4] S. Chen, W. Li, J. Wu, Q. Jiang, M. Tang, S. Shutts, S.N. Elliott, A. Sobiesierski, A.J. Seeds, I. Ross, P.M. Smowton, H. Liu. Nature Photonics, 10, 307 (2016).

[5] Y. Wan, J. Norman, Q. Li, M.J. Kennedy, D. Liang, C. Zhang, D. Huang, Z. Zhang, A.Y. Liu, A. Torres, D. Jung, A.C. Gossard, E.L. Hu, K.M. Lau, J.E. Bowers. Optica, 4, 940 (2017).

[6] N. Kryzhanovskaya, E. Moiseev, Yu. Polubavkina, M. Maximov, M. Kulagina, S. Troshkov, Yu. Zadiranov, Yu. Guseva, A. Lipovskii, M. Tang, M. Liao, J. Wu, S. Chen, H. Liu, A. Zhukov. Optics Lett., 42, 3319 (2017).

[7] A. Gubenko, D. Livshits, I. Krestnikov, S. Mikhrin, A. Kozhukhov, A. Kovsh, N. Ledentsov, A. Zhukov, E. Portnoi. Electron. Lett., 41, 1124 (2005).

[8] Y. Ben-Ezra, B.I. Lembrikov. Quantum Dot-Semiconductor Optical Amplifiers (QDSOA): Dynamics and Applications. In: Optical Amplifiers - A Few Different Dimensions (ed. by P.K. Choudhury), Ch. 2 (IntechOpen, 2018).

[9] M.V. Maximov, L.V. Asryan, Yu.M. Shernyakov, A.F. Tsatsul'nikov, I.N. Kaiander, V.V. Nikolaev, A.R. Kovsh, S.S. Mikhrin, V.M. Ustinov, A.E. Zhukov, Zh.I. Alferov, N.N. Ledentsov, D. Bimberg. IEEE J. Quant. Electron., 37, 676 (2001).

[10] Y. Ben-Ezra, M. Haridim, B.I. Lembrikov. IEEE Photon. Technol. Lett., 17, 1803 (2005).

[11] M. Shojaei-Oghani, M.H. Yavari. Optical Quant. Electron., 50, 374 (2018).

[12] F. Hakimian, M.R. Shayesteh, M. Moslemi. J. Optoelectron. Nanostruct., 4, 1 (2019).

[13] T.W. Berg, J. Mørk. IEEE J. Quant. Electron., 40, 1527 (2004).

[14] L.A. Coldren, S.W. Corzine, M.L. Mašanović. Photonic Integrated. Circuits. In: Diode Lasers and Photonic Integrated Circuits. 2nd edn (ed. by K. Chang) Ch. 8 (Hoboken: Wiley, 2012).

[15] A.E. Zhukov, A.R. Kovsh, V.M. Ustinov, A.Yu. Egorov, N.N. Ledentsov, A.F. Tsatsul'nikov, M.V. Maximov, Yu.M. Shernyakov, V.I. Kopchatov, A.V. Lunev, P.S. Kop'ev, D. Bimberg, Zh.I. Alferov. Semicond. Sci. Technol., 14, 118 (1999).

[16] A.E. Zhukov, A.R. Kovsh, D.A. Livshits, V.M. Ustinov, Zh.I. Alferov. Semicond. Sci. Technol., 18, 774 (2003).

[17] A. Markus, A. Fiore. Phys. Status Solidi A, 201, 338 (2004).

[18] L.V. Asryan, Y. Wu, R.A. Suris. Appl. Phys. Lett., 98, 131108 (2011).

[19] V. Talalaev, N. Kryzhanovskaya, J.W. Tomm, V. Rutckaia, J. Schilling, A. Zhukov. Sci. Rep., 9, 5635 (2019).

[20] V.V. Korenev, A.V. Savelyev, A.E. Zhukov, A.V. Omelchenko, M.V. Maximov. Appl. Phys. Lett., 102, 112101 (2013).

[21] V.V. Korenev, A.V. Savelyev, M.V. Maximov, F.I. Zubov, Yu.M. Shernyakov, M.M. Kulagina, A.E. Zhukov. Appl. Phys. Lett., 111, 132103 (2017).

Редактор Г.А. Оганесян 


\title{
Saturation power of semiconductor optical amplifier based on self-organized quantum dots
}

A.E. Zhukov' ${ }^{1}$, N.V. Kryzhanovskaya ${ }^{1}$, E.I. Moiseev', A.M. Nadtochiy ${ }^{1}$, F.I. Zubov' ${ }^{2}$, M.V. Fetisova ${ }^{2}$, M.V. Maximov ${ }^{2,1}$, N.Yu. Gordeev ${ }^{3}$

${ }^{1}$ National Research University Higher School of Economics, 190008 St. Petersburg, Russia

${ }^{2}$ Alferov St. Petersburg Academic University, 194021 St. Petersburg, Russia

3 loffe institute,

194021 St. Petersburg, Russia

\begin{abstract}
Gain saturation in a semiconductor optical amplifier with an array of quantum dots was studied analytically and by numerical simulation on the basis of an analysis of the rate equations. It is shown that, at a moderate injection level, the saturation power increases in proportion to the current density, and then reaches its maximum value, limited by the rate of capture of charge carriers to the ground state and by the number of quantum dots interacting with photons. Expressions are proposed that allow an explicit description of the dependence of the saturation power on the current and its relationship with the internal parameters of the active region.
\end{abstract}

